\title{
A blind EM-based carrier frequency offset estimation algorithm for OFDM signals
}

\author{
Yanyan Huang , Hua Peng, Non-members \\ Information Engineering University, Zheng Zhou, He Nan \\ [e-mail: 18638575039@163.com] \\ *Corresponding author: Yanyan Huang
}

Keywords: OFDM, carrier frequency offset, blind, EM, second-order cyclostationarity

\begin{abstract}
Orthogonal frequency-division multiplexing (OFDM) system is very sensitive to the synchronization errors in digital communications, which should be compensated at the receiver. In this paper, we proposed an expectation maximum (EM) based blind carrier frequency offset (CFO) estimation algorithm for OFDM signals. By utilizing the second-order cyclostationarity of OFDM signals, a blind frequency offset estimation method is introduced as the initialization of EM algorithm. Without any prior information of the received signal, this method can realize the blind CFO estimation of OFDM after a few iterative process. Simulation results show that the proposed algorithm can effectively estimate the CFO at moderate and high signal to noise rate (SNR).
\end{abstract}

\section{Introduction}

OFDM has been widely recognized as an efficient transmission technique for wireless communications [1]. In OFDM systems, each subcarrier has a bandwidth narrow enough to experience flat fading, which makes the signal robust against a frequency selective fading channel. However, OFDM systems are known to be sensitive to CFO, which destroys the orthogonal property among subcarriers and induces intercarrier interference (ICI). ICI also complicates the data detection. Therefore, frequency synchronization becomes a critical part in the design of OFDM receivers [2].

Several frequency estimation schemes for OFDM applications have been investigated. References [3-10] provide a good sample of the results obtained in this area. In particular, the method reported in [3] gives the maximum likelihood (ML) estimator of the frequency offset, based on the observation of two consecutive and identical symbols. Reference [4] exploits the redundancy associated with cyclic prefix in the OFDM symbols. But this kind of method can only get the coarse estimation result. The fine result can be get through expectation-maximization (EM) algorithm.

EM-based method is a good kind of iterative method, which has been widely used in most areas of digital communications. There are kinds of EM methods to estimate the CFO of OFDM which need an initial step as a start of iterative process [5-8]. In [5], the authors propose an iterative CFO estimator for OFDM systems impaired by narrow band interference. [6] gives a semiblind estimation algorithm under unknown double selective channels. This method takes ML to initialize the CFO and channel estimation, then iterates the CFO、 channel estimation and data detection using limited pilot subcarriers. References [7] and [8] aim at the MIMO-OFDM system. Particularly, [7] takes ML method to estimate the CFO. In [8] the LDPC decoding result is incorporated to iterative process.

It can be seen that the initial step of the EM-based methods are pilot aided or depend on training sequences in existing methods. However, it is difficult to get the training sequence in the non-cooperative communication. So the blind CFO estimation is necessary. In this paper, a new blind CFO estimation algorithm along with the blind initial step is proposed. Here, we take the correlation based method[9] as the initial estimation. This method relies on second-order statistics only and exploits the cyclostationarity of the OFDM signal.

This paper is organized as follows. In Section 2, the pulse shaping OFDM signal model is given and we provide the problem statement. Section 3 introduces the new estimators and discusses their 
properties. Section 4 presents simulation results, and Section 5 concludes the paper.

\section{Signal model}

The baseband equivalent of a pulse shaping OFDM signal is given by

$$
\mathrm{s}(\mathrm{n})=\sum_{k=0}^{N-1} \sum_{\mathrm{l}=-\infty}^{\infty} \mathrm{x}_{\mathrm{k}, \mathrm{l}} \mathrm{w}(\mathrm{k}) \mathrm{g}(\mathrm{n}-\mathrm{lL}) \mathrm{e}^{\mathrm{j}(2 \pi / \mathrm{N}) \mathrm{k}(\mathrm{n}-\mathrm{lM})}
$$

where $\mathrm{N}$ is the number of subcarriers, $\mathrm{L}$ is the symbol length, $\mathrm{g}[\mathrm{n}]$ denotes the transmitter pulse shaping filter, and $\mathrm{x}_{\mathrm{k}, \mathrm{l}}$ denotes the data symbols (taken from a finite complex alphabet constellation). $\mathrm{w}(\mathrm{k})$ is the subcarrier weighting, that is the individual subcarriers are transmitted with different powers. We assume that the receiver knows the pulse shaping filter $g(n)$, the subcarriers weighting $\mathrm{w}(\mathrm{k})$ and the variances $\sigma_{\mathrm{x}}^{2}$.

Then a cyclic prefix (CP) is inserted at the beginning of each OFDM symbol to prevent inter-symbol interference (ISI). The CP added signal is then transmitted through an additive white Gaussian noise (AWGN) channel with carrier frequency offsets. At the receiver, assuming perfect timing synchronization is achieved, the nth sample of the received signal is given by

$$
\mathrm{y}(\mathrm{n})=\mathrm{e}^{\mathrm{j} 2 \pi \varepsilon \mathrm{n} / \mathrm{N}} \mathrm{s}(\mathrm{n})+\rho(\mathrm{n})
$$

where $\varepsilon$ denotes the carrier frequency offset as a fraction of the subcarrier spacing. Notice that all subcarriers experience the same $\varepsilon$ in one OFDM symbol. $\rho(n)$ is the zero mean additive white Gaussian noise with power $\sigma^{2}$. A block diagram of the transceiver is shown in Figure 1.

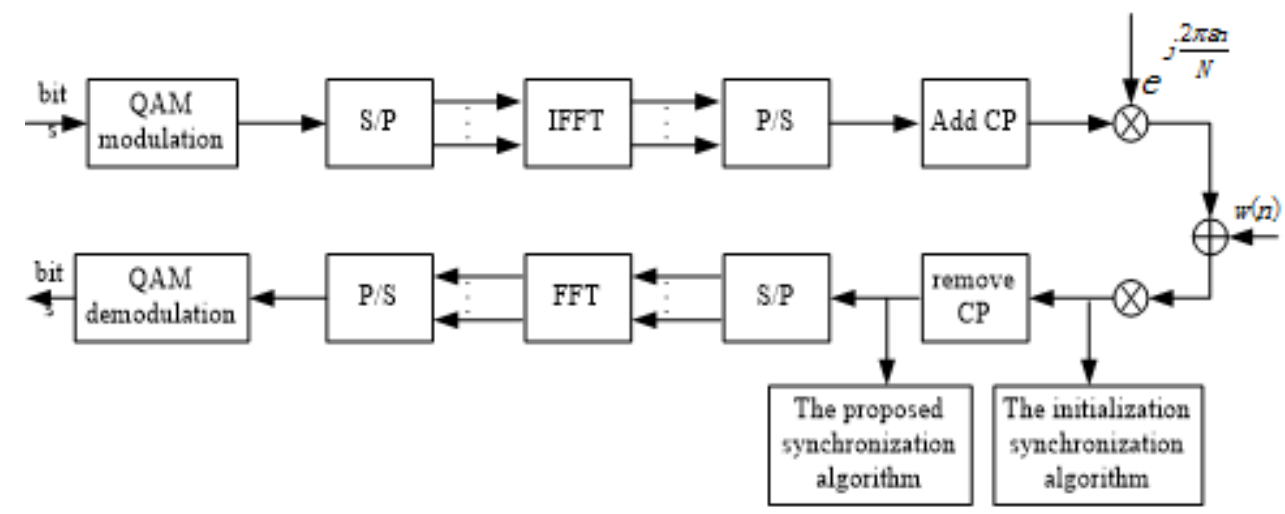

Fig. 1 Transceiver structure of an OFDM system

After discarding the $C P$, the received signal $y=[y(0), \ldots, y(N-1)]^{T}$ for a whole OFDM symbol can be expressed in a vector form as

$$
\begin{aligned}
\mathbf{y} & =\boldsymbol{\Phi}(\varepsilon) \mathbf{s}+\boldsymbol{\rho} \\
& =\boldsymbol{\Phi}(\varepsilon) \mathbf{F}^{H} \mathbf{x}+\boldsymbol{\rho}
\end{aligned}
$$

Where $\Phi(\varepsilon)=\operatorname{diag}\left\{1, \mathrm{e}^{\mathrm{j} 2 \pi \varepsilon / \mathrm{N}}, \ldots, \mathrm{e}^{\mathrm{j} 2 \pi \varepsilon(\mathrm{N}-1) / \mathrm{N}}\right\}, \rho=[\rho(0), \ldots, \rho(\mathrm{N}-1)]^{\mathrm{T}} . \mathrm{F}$ is the FFT matrix

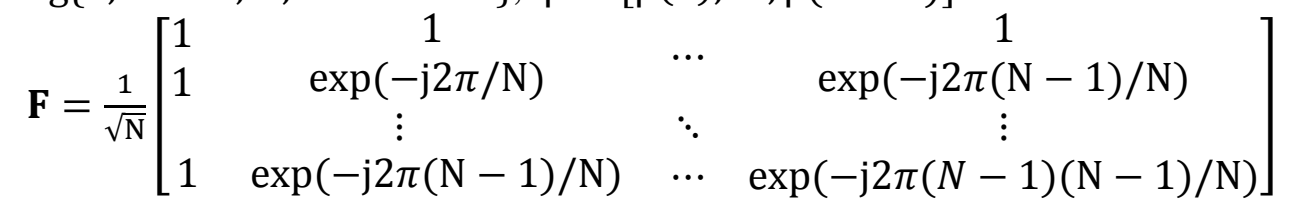

After taking FFT, the signal is

$$
\mathbf{Y}=\mathbf{F} \boldsymbol{\Phi}(\varepsilon) \mathbf{F}^{H} \mathbf{x}+\boldsymbol{\rho}
$$

Here the received signal is partitioned into $\mathrm{K}$ adjacent segments, each composed of $\mathrm{M}$ elements and corresponding to one of the repetitive parts in which the block is divided. Every segment is identified by a time index $\mathrm{k}$, with $\mathrm{k}=0,1, \ldots, \mathrm{K}-1$, and is passed to an M-point DFT unit. We denote by $\mathrm{Y}(\mathrm{m}, \mathrm{k})$ the mth DFT output of the kth segment and arrange all these quantities into $\mathrm{M}$ vectors $\mathrm{Y}(\mathrm{m})=[\mathrm{Y}(\mathrm{m}, 0), \mathrm{Y}(\mathrm{m}, 1), \ldots, \mathrm{Y}(\mathrm{m}, \mathrm{K}-1)]^{\mathrm{T}}$ with $\mathrm{m}=0,1, \ldots, \mathrm{M}-1$, and we can get

$\mathrm{Y}(\mathrm{m})=\mathrm{S}(\mathrm{m}) \mathrm{e}_{\mathrm{K}}(\varepsilon)+\rho(\mathrm{m})$

Where $\mathrm{e}_{\mathrm{K}}(\varepsilon)=\left[1, \mathrm{e}^{\mathrm{j} 2 \pi \varepsilon / \mathrm{K}}, \mathrm{e}^{\mathrm{j} 4 \pi \varepsilon / \mathrm{K}}, \ldots, \mathrm{e}^{\mathrm{j} 2 \pi(\mathrm{K}-1) \varepsilon / \mathrm{K}}\right]^{\mathrm{T}}$ collects the phase shifts introduced by the 
CFO. $\mathrm{S}(\mathrm{m})$ is the signal component.

\section{The proposed method}

\subsection{EM approach}

EM is a general method of determining the ML estimate of parameters of the underlying distribution from a given set of data which is incomplete[10].

Here we view $\mathrm{Y}=\left[\mathrm{Y}^{\mathrm{T}}(0), \mathrm{Y}^{\mathrm{T}}(1), \ldots, \mathrm{Y}^{\mathrm{T}}(\mathrm{M}-1)\right]^{\mathrm{T}}$ as the incomplete data and define the complete data set as the pair $\left(\mathrm{Y}, \sigma^{2}\right)$. Also, we denote $\theta=(\varepsilon, S)$ the parameters to be estimated and call $\hat{\theta}_{\mathrm{i}}=\left(\hat{\varepsilon}_{\mathrm{i}}, \widehat{S}_{\mathrm{i}}\right)$ their estimate at the ith iteration, where $\sigma^{2}=\left[\sigma^{2}(0), \sigma^{2}(1), \ldots, \sigma^{2}(\mathrm{M}-1)\right]^{\mathrm{T}}$ and $S=[S(0), S(1), \ldots, S(M-1)]^{T}$. EM algorithm iteratively alternates between an E-step, calculating the expectation of the log-likelihood function of the complete data given the observations and the current estimate $\hat{\theta}_{\mathrm{i}}$, and an $\mathrm{M}-\mathrm{step}$, maximizing that expectation with respect to the unknown parameters. The E-step is

Where $\widetilde{\boldsymbol{\theta}}=(\widetilde{\varepsilon}, \widetilde{\mathbf{S}})$ is a trial value of $\boldsymbol{\theta}$.

$$
Q\left(\widetilde{\boldsymbol{\theta}} \mid \widehat{\boldsymbol{\theta}}_{i}\right)=\mathrm{E}_{\boldsymbol{\sigma}^{2}}\left\{\ln \left[\mathrm{p}\left(\mathbf{Y} \mid \boldsymbol{\sigma}^{2}, \widetilde{\boldsymbol{\theta}}\right)\right] \mathrm{p}\left(\mathbf{Y} \mid \boldsymbol{\sigma}^{2}, \widehat{\boldsymbol{\theta}}_{\mathbf{i}}\right)\right\}
$$

The M-step is

$$
\widehat{\boldsymbol{\theta}}_{i+1}=\operatorname{argmax}_{\widetilde{\theta}}\left\{Q\left(\widetilde{\boldsymbol{\theta}} \mid \widehat{\boldsymbol{\theta}}_{i}\right)\right\}
$$

The derivations of the E-step and M-step are detailed as follows.

\section{E-step:}

After skipping irrelevant factors and additive terms, function $Q\left(\widetilde{\boldsymbol{\theta}} \mid \widehat{\boldsymbol{\theta}}_{i}\right)$ can be equivalently replaced by

$$
\Lambda\left(\widetilde{\boldsymbol{\theta}} \mid \widehat{\boldsymbol{\theta}}_{i}\right)=-\frac{1}{K} \sum_{m=0}^{M-1} \frac{1}{\hat{\sigma}_{i}^{2}(m)}\left\|\mathbf{X}(m)-\tilde{\mathbf{S}}(m) \mathbf{e}_{\mathrm{K}}(\widetilde{\varepsilon})\right\|^{2}
$$

Where $\|\cdot\|$ denotes the Euclidean norm of the enclosed vector and $\hat{\sigma}_{i}^{2}$ represents a biased estimate of $\sigma^{2}(m)$ at the ith iteration.

\section{M-step:}

$$
\hat{\sigma}_{i}^{2}(m)=\frac{1}{K}\left[\lambda+\left\|\mathbf{X}(m)-\hat{S}_{i}(m) \mathbf{e}_{\mathrm{K}}\left(\hat{\varepsilon}_{i}\right)\right\|^{2}\right]
$$

Maximizing $\Lambda\left(\widetilde{\boldsymbol{\theta}} \mid \widehat{\boldsymbol{\theta}}_{i}\right)$ with respect to $\tilde{\varepsilon}$ and $\tilde{\boldsymbol{S}}$ produces

$$
\hat{\theta}_{i+1}=\operatorname{argmax}_{\widetilde{\boldsymbol{\theta}}}\left\{\Lambda\left(\widetilde{\boldsymbol{\theta}} \mid \widehat{\boldsymbol{\theta}}_{i}\right)\right\}
$$

$$
\widehat{\varepsilon}_{i+1}=\underset{\tilde{\varepsilon}}{\operatorname{argmax}}\left\{\Gamma_{i}(\widetilde{\varepsilon})\right\}
$$

With

$$
\Gamma_{i}(\tilde{\varepsilon})=\sum_{m=0}^{M-1} \frac{1}{\widehat{\sigma}_{i}^{2}(m)}\left|\mathbf{e}_{\mathrm{K}}^{\mathrm{H}}(\widehat{\varepsilon}) \mathbf{X}(\mathrm{m})\right|^{2}
$$

and

$$
\hat{S}_{i+1}(m)=\frac{1}{K} \mathbf{e}_{\mathrm{K}}^{\mathrm{H}}\left(\hat{\varepsilon}_{\mathrm{i}+1}\right) \mathbf{X}(\mathrm{m})
$$

Based on the approach suggested in [11], which allows one to compute $\hat{\varepsilon}_{i+1}$ in closed-form without resorting to any peak search procedure, we can rewrite the right-hand-side of (13) in the form

where

$$
\Gamma_{i}(\widetilde{\varepsilon})=\operatorname{Re}\left\{\sum_{k=0}^{K-1} R_{i}(k) e^{-j 2 \pi k \tilde{\varepsilon} / K}\right\}
$$

$$
R_{i}(k)=\sum_{m=0}^{M-1} \frac{\gamma(m, k)}{\widehat{\sigma}_{i}^{2}(m)}
$$

And

$$
\gamma(m, k)=\sum_{p=k}^{K-1} X(m, p) X^{*}(m, p-k)
$$

$\gamma(m, k)$ is the k-lag sample correlation function over the $m$ th subcarrier. 
The maximization of $\Gamma_{i}(\widetilde{\varepsilon})$ is performed by two steps, including a coarse step and an iterative step. In the coarse step, we introduce a blind method based on the second-order cyclostationarity which will be detailed as follows. Assume the coarse estimate of the CFO is $\hat{\varepsilon}_{i+1}^{(c)}$, and it is subsequently refined in the second step by looking for an estimate of the residual error $\Delta \varepsilon=\varepsilon-$ $\widehat{\varepsilon}_{i+1}^{(c)}$. For this purpose, we let

And rewrite (15) in the equivalent form

$$
R_{i}^{(c)}(k)=R_{i}(k) e^{-j 2 \pi k \widehat{\varepsilon}_{i+1}^{(c)} / K}
$$

$$
\Gamma_{i}(\Delta \tilde{\varepsilon})=\operatorname{Re}\left\{\sum_{\mathrm{k}=0}^{\mathrm{K}-1} \mathrm{R}_{\mathrm{i}}^{(\mathrm{c})}(\mathrm{k}) \mathrm{e}^{-\mathrm{j} 2 \pi \mathrm{k} \Delta \tilde{\varepsilon} / \mathrm{K}}\right\}
$$

With $\Delta \widetilde{\varepsilon}=\tilde{\varepsilon}-\widehat{\varepsilon}_{i+1}^{(c)}$. Setting to zero the derivative of (19) with respect to $\Delta \widetilde{\varepsilon}$ and assuming that $\Delta \tilde{\xi}$ is small enough, an estimate of $\Delta \xi$ at the (i+1)th iteration is given by

$$
\Delta \widehat{\varepsilon}_{i+1}=\frac{\mathrm{K}}{2 \pi} \frac{\sum_{\mathrm{k}=1}^{\mathrm{K}} \mathrm{k} \cdot \operatorname{Im}\left\{\mathrm{R}_{\mathrm{i}}^{(\mathrm{c})}(\mathrm{k})\right\}}{\sum_{\mathrm{k}=1}^{\mathrm{K}} \mathrm{k}^{2} \cdot \operatorname{Re}\left\{\mathrm{R}_{\mathrm{i}}^{(\mathrm{c})}(\mathrm{k})\right\}}
$$

The CFO estimate is eventually computed as

And the signal estimatation is get as (14) shows.

$$
\widehat{\varepsilon}_{i+1}=\Delta \widehat{\varepsilon}_{i+1}+\widehat{\varepsilon}_{i+1}^{(c)}
$$

\subsection{Initialization step: Blind CFO estimation based on the second-order cyclic cumulant}

A good initialization is essential to EM algorithm. In the non-cooperative communication system, the pilot sequence is hard to get. So a blind parameter estimation algorithm is needed in the receiver. Here, we use the high-order cyclic cumulants to get the blind CFO estimation. Then this CFO estimation is incorporated into the initialization step of the iterative receiver.

The highlight feature of digital communication signals is that they have cyclostationarity, the cyclic cumulants has been an effective signal processing tool for analyzing the digital communication signals. Theoretically, the high-order cyclic cumulants can suppress any noises of stationary Gaussian、non- Gaussian or non- stationary Gaussian, so high signal-to-noise can be get in the area of high-order cyclic cumulants, which is benefit for the parameter estimation.

The correlation function of a stochastic process is defined as $c_{y}[n, \tau]=E\left\{y(n) y^{*}(n-\tau)\right\}$, where $\tau$ is an integer lag parameter. The signal $y(n)$ is said to be second-order cyclostationary (CS) with period $L$ if $c_{y}[n, \tau]=c_{y}[n+L, \tau]$. The correlation function of the received OFDM signal $y(n)$ is given by

$$
\mathrm{c}_{\mathrm{y}}[n, \tau]=\sigma_{x}^{2} \Gamma_{N}[\tau] \sum_{l=-\infty}^{\infty} g[n-l L] g[n-l L-\tau]+c_{\rho}[\tau]
$$

Here

$$
\Gamma_{N}[\tau]=\sum_{\mathrm{k}=0}^{\mathrm{N}-1}|\mathrm{w}(\mathrm{k})|^{2} \mathrm{e}^{\mathrm{j}(2 \pi / \mathrm{N}) \mathrm{k} \tau}
$$

is the N-point inverse discrete Fourier transform (DFT) of $|w(k)|^{2}$.

For the general case of OFDM with time-frequency guard regions, cyclostationarity is caused by the time-frequency guard regions and by the overlapping nature of the pulse shaping filter. If subcarrier weighting is employed, $c_{y}[n, \tau]$ will contain information on the synchronization parameters for all $\tau$.

For a fixed lag $\tau, c_{y}[n, \tau]$ can therefore be expanded into a Fourier series with Fourier coefficients given by

$$
C_{y}[k, \tau]=\frac{1}{L} \sum_{n=0}^{L-1} c_{y}[n, \tau] e^{-j(2 \pi / L) k n}, \mathrm{k}=0,1, \ldots, \mathrm{L}-1
$$

After derivation, $C_{y}[k, \tau]$ can be expressed as

$$
C_{y}[k, \tau]=\frac{\sigma_{x}^{2}}{L} e^{j 2 \pi \varepsilon \tau} \Gamma_{N}[\tau] A^{(g, g)}\left[\tau, \frac{k}{L}\right)+c_{\rho}[\tau] \delta[k], k=0,1, \ldots, L-1
$$

Since $\sigma_{\mathrm{x}}^{2}, \sigma_{\mathrm{x}, \mathrm{R}}^{2}, \sigma_{\mathrm{x}, \mathrm{I}}^{2} \mathrm{I}[\mathrm{n}], \mathrm{w}[\mathrm{k}]$, and $\Gamma_{\mathrm{N}}[\tau]$ are known in the receiver, their influence can be eliminated by defining 


$$
\mathrm{C}[\mathrm{k}, \tau]=\left\{\begin{array}{lc}
\frac{C_{y}[k, \tau]}{\frac{\sigma_{x}^{2}}{M} \Gamma_{N}[\tau] A^{(g, g)}\left[\tau, \frac{k}{L}\right)}, & {[k, \tau] \in \mathcal{L}} \\
0, & \text { else }
\end{array}\right.
$$

Where $\mathcal{L}:=\left\{[k, \tau] \mid \Gamma_{N}[\tau] A^{(g, g)}\left[\tau, \frac{k}{L}\right) \neq 0\right\} . A^{(g, g)}\left[\tau, \frac{k}{L}\right)=\sum_{n=-\infty}^{\infty} g(n) g(n-\tau) e^{-j 2 \pi n \frac{k}{L}}$. Then the coarse carrier frequency offset estimation can be retrieved from $\mathrm{C}[\mathrm{k}, \tau]$,

$$
\varepsilon_{i+1}^{(c)}=\frac{\arg \{\mathrm{C}[\mathrm{k}, \tau] \mathrm{C}[\mathrm{L}-\mathrm{k}, \tau]\}}{4 \pi \tau}, \quad[k, \tau] \in \mathcal{L}
$$

in practice, the cyclic statistics $C_{y}[k, \tau]$ can be estimated from a finite data record $\{y[n]\}_{n=0}^{L-1}$ of length $\mathrm{L}$ according to

Then we have

$$
\hat{C}_{y}[k, \tau]=\frac{1}{L} \sum_{n=0}^{L-1} y[n] y^{*}[n-\tau] e^{-j(2 \pi / L) k n}
$$

$$
\widehat{\varepsilon}_{i+1}^{(c)}=\frac{1}{4 \pi\left|\mathcal{L}^{\prime}\right|} \sum_{[k, \tau] \in \mathcal{L}^{\prime}} \frac{1}{\tau} \arg \{\hat{C}[k, \tau] \hat{C}[L-k, \tau]\}
$$

Where $\hat{C}[k, \tau]$ is obtained by replacing $C_{y}[k, \tau]$ in (26) by $\hat{C}_{y}[k, \tau], \mathcal{L}^{\prime}=\mathcal{L}\{k=0\}\{\tau=0\}$.

\section{Simulation results}

In this section, we provide simulation results demonstrating the performance of the proposed estimators. We simulated a pulse shaping OFDM/QAM system with time-frequency guard region, $\mathrm{N}=8$ channels, symbol length $\mathrm{M}=16$. All results were obtained by averaging over 200 independent Monte Carlo trials. Each realization consisted of 1024 data symbols. The subchannel

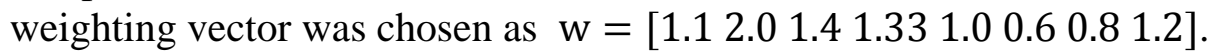

Fig. 2 shows the frequency MSE as a function of SNR. The number of iterations varies from 1 to 3 while $\mathrm{K}$ is set to $4, \varepsilon=0.0625$.

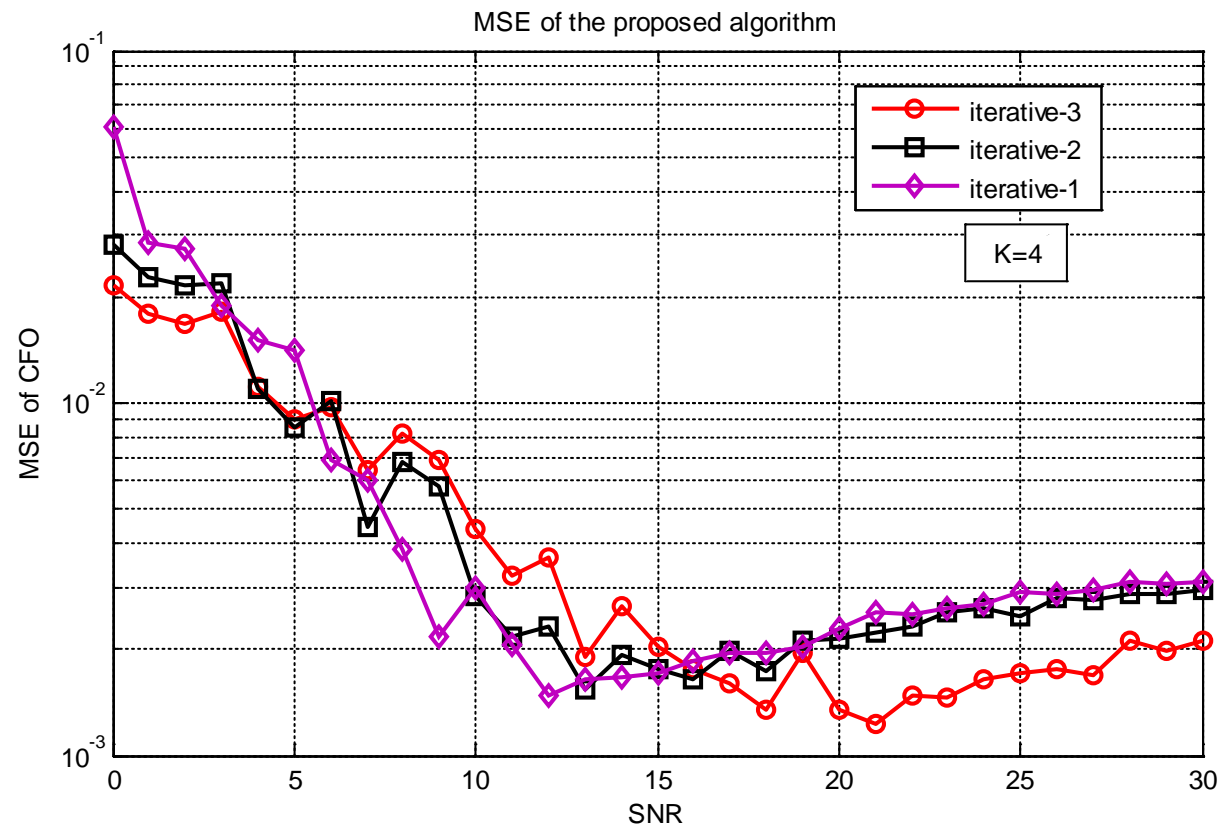

Fig.2 Accuracy of the frequency estimators and $\mathrm{K}=4$ 


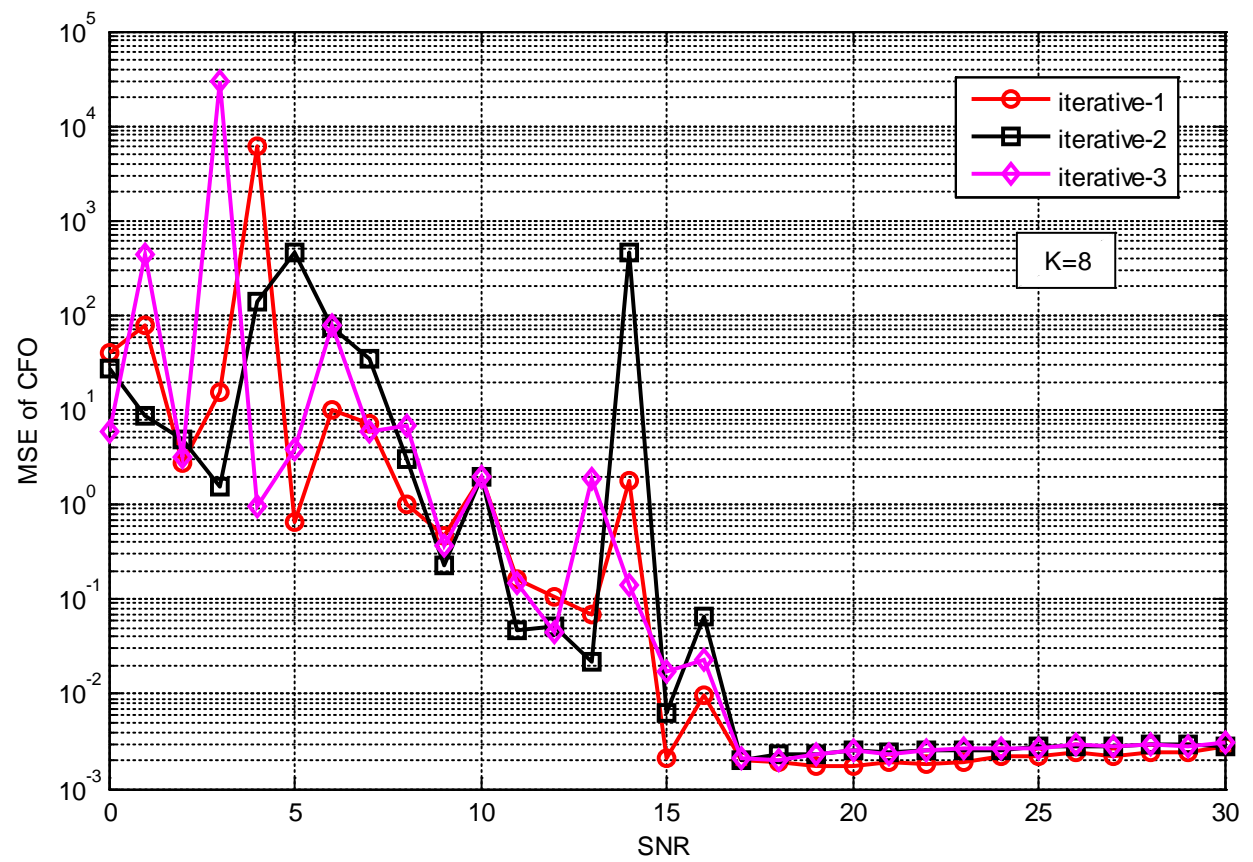

Fig. 3 Accuracy of the frequency estimators and $\mathrm{K}=8$

The results of Fig.3 are obtained in the same operating conditions of Fig.2 except for the value of $\mathrm{K}$, which is set to 8. Compared with Fig.3, it turns out that the performance of the frequency estimators improves with $\mathrm{K}$ at moderate and high SNR.

In Fig. 4, we computed the MSE of the CFO estimator for the AWGN case and in the presence of an unknown four-tap multipath channel.

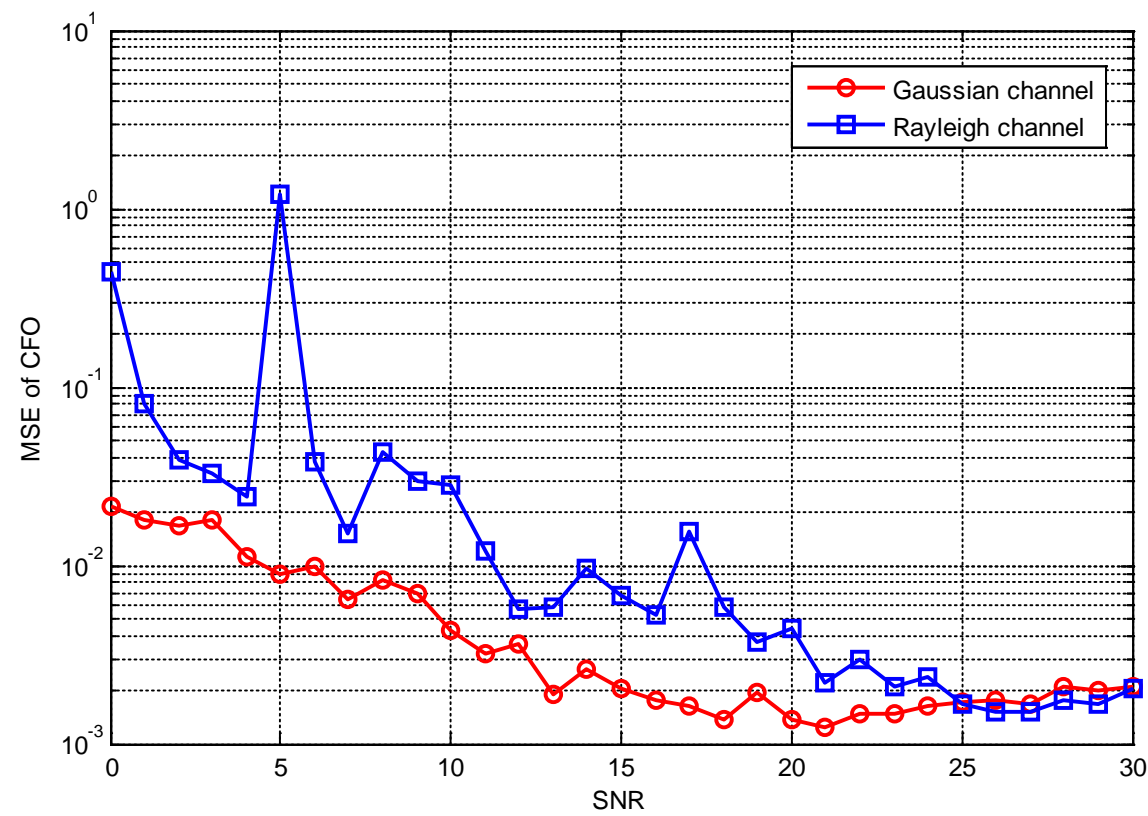

Fig.4 MSE of the CFO in the AWGN case and the multipath case

In the AWGN case, our estimators perform well at all SNR values. Also, we can see that the presence of an unknown multipath channel leads to a degradation in the estimator performance.

Fig. 5 computed the MSE of CFO at an SNR of $9 \mathrm{~dB}$ as a function of the carrier frequency offset. Fig. 5 shows that the performance of the estimator improves for smaller values of $\varepsilon$. 


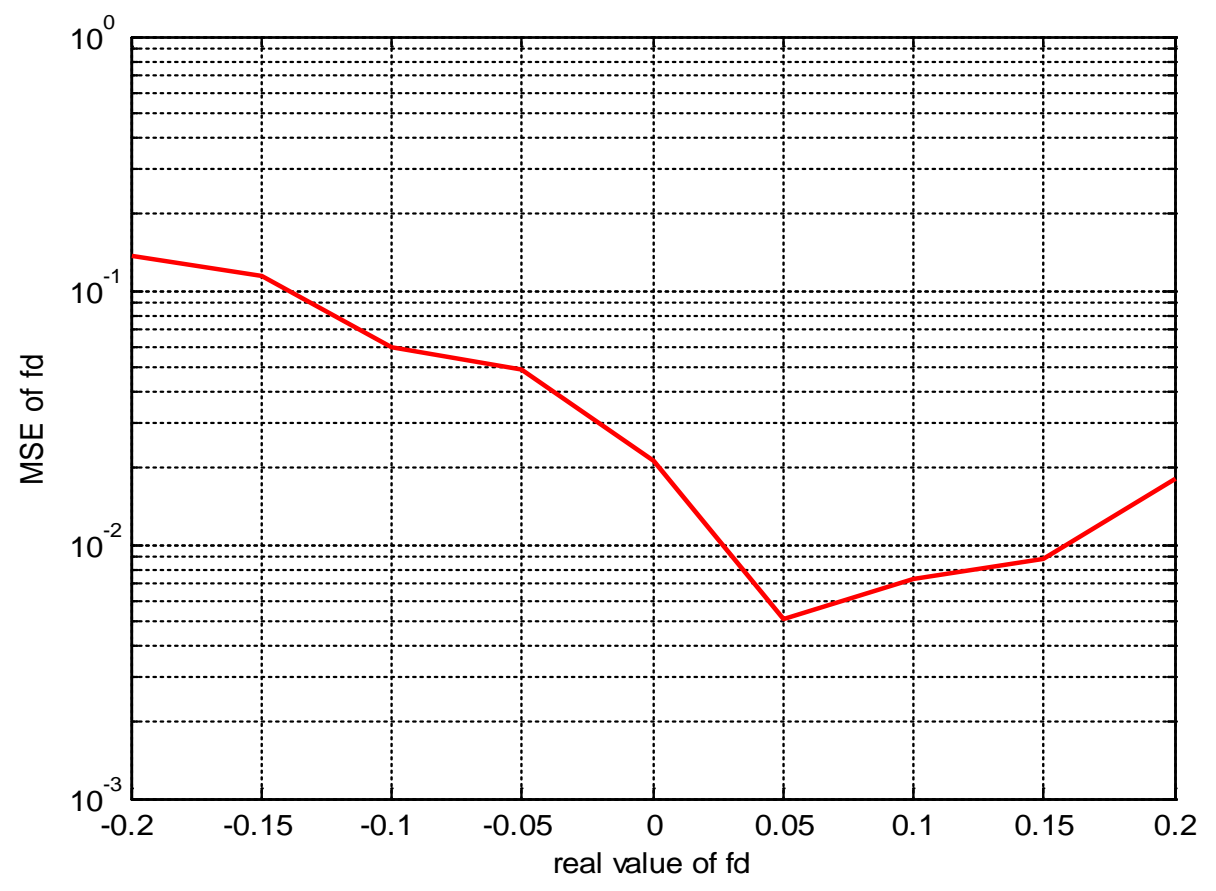

Fig.5 MSE of fd versus fd as a function of $\mathrm{fd}$

\section{Conclusions}

In this paper, we proposed a total blind CFO estimation method of OFDM signals, which utilize the second-order cyclostationarity and the EM iterative method. Different from existing EM based methods, this method gives a blind initial process, which can be used in the non-cooperative communications. Simulation results proves that this method can estimate the CFO at moderate and high SNR.

\section{References}

[1] A.Peled and A.Ruiz, "Frequency domain data transmission using reduced computational complexity algorithms," in Proc. of IEEE International Conference on Acoustics, Speech, and Signal Processing, pp. 964-967, Apr, 1980.

[2] T.Pollet and M.Moeneclaey, "The effect of carrier frequency offset on the performance of band limited single carrier and OFDM signals," in Proc. of IEEE Global Telecommunications Conference, pp.719-723, Nov.18-22, 1996.

[3] P.H.Moose, "A Technique for Orthogonal Frequency Division Multiplexing Frequency Offset Correction,” IEEE Trans. On Communications, vol. 42, no.10, pp.2908-2914, Oct. 1994.

[4] J.J. van de Beek, M.Sandell, P.O.Borjesson, "ML Estimation of Time and Frequency Offset in OFDM systems,” IEEE Trans. on signal processing, vol. 45, no.7, pp.1800-1805, July, 1997.

[5] Luca Sanguinetti, Michele Morelli, "An EM-based frequency offset estimator for OFDM systems with unknown interference," IEEE Trans. on wireless communications, vol.8, no.9, pp:4470-4475, September, 2009.

[6] Lanlan He, Shaodan Ma, Yik-Chung Wu and Tung-Sang Ng. "Semiblind Iterative Data Detection for OFDM Systems with CFO and Doubly Selective Channels”, IEEE Trans. on communications, vol. 58, no.12, pp. 3491-3499, December 2010.

[7] Yong Sun, Zixiang Xiong, and Xiaodong Wang, "EM-Based Iterative Receiver Design With Carrier-Frequency Offset Estimation for MIMO OFDM Systems," IEEE Trans. on communications, vol.53, no.4, pp. 581-586, April 2005.

[8] S. Salari, M. Ahmadian, and M. Ardebilipour , "Carrier-Frequency Offset Estimation via the EM Algorithm for MIMO-OFDM Iterative Receivers," in Proc. of IEEE Fifth Annual Conference on 
Communication Networks and Services Research, pp:101-106, May 14-17, 2007.

[9] Bolcskei, Helmut, "Blind estimation of symbol timing and carrier frequency offset in wireless OFDM systems,” IEEE Trans. on communications, vol. 49, no.6, pp:988-999, Jun, 2001.

[10] A.P.Dempster, N.M.Laird, and D.R.Rubin, "Maximum likelihood from incomplete data via EM algorithm,” J.Royal Stat. Soc. vol.39, pp.1-38,1977.

[11] M.Ghogho, A.Swami, and P.Ciblat, "Training design for CFO estimation in OFDM over correlated multipath fading channels," in Proc. of IEEE Global Telecommunications Conference, pp.2821-2825, Nov.2007.

Yanyan Huang is a doctoral student at the information engineering university in zheng zhou, He nan. Her research interests are carrier synchronization of wireless communications, channel estimation and equalization.

Hua Peng is a professor at the information engineering university in zheng zhou, He nan. His research interests are signal professing in wireless communications. 